\title{
Benign Ovarian Serous Tumor
}

National Cancer Institute

\section{Source}

National Cancer Institute. Benign Ovarian Serous Tumor. NCI Thesaurus. Code C7313.

A non-metastasizing neoplasm that arises from the ovary and is characterized by the presence of neoplastic epithelium that resembles the epithelium of the fallopian tube. It includes serous cystadenoma, serous adenofibroma, serous cystadenofibroma, and surface papilloma. 